\title{
THE FIXED POINT FOR A TRANSFORMATION OF HAUSDORFF MOMENT SEQUENCES AND ITERATION OF A RATIONAL FUNCTION
}

\author{
CHRISTIAN BERG and ANTONIO J. DURÁN*
}

\begin{abstract}
We study the fixed point for a non-linear transformation in the set of Hausdorff moment sequences, defined by the formula: $T\left(\left(a_{n}\right)\right)_{n}=1 /\left(a_{0}+\cdots+a_{n}\right)$. We determine the corresponding measure $\mu$, which has an increasing and convex density on $] 0,1[$, and we study some analytic functions related to it. The Mellin transform $F$ of $\mu$ extends to a meromorphic function in the whole complex plane. It can be characterized in analogy with the Gamma function as the unique log-convex function on ] $-1, \infty$ [ satisfying $F(0)=1$ and the functional equation $1 / F(s)=1 / F(s+1)-F(s+1)$, $s>-1$.
\end{abstract}

\section{Introduction and main results}

Hausdorff moment sequences are sequences of the form $\int_{0}^{1} t^{n} d v(t), n \geq 0$, where $v$ is a positive measure on $[0,1]$. Hausdorff moment sequences were characterized as completely monotonic sequences in a fundamental paper by Hausdorff, see [17]. For a recent study of Hausdorff moment sequences see [14], [15]. Hausdorff moment sequences can also be characterized as bounded Stieltjes moment sequences, where Stieltjes moment sequences are of the form $\int_{0}^{\infty} t^{n} d v(t), n \geq 0$ for a positive measure $v$ on $[0, \infty[$. For a treatment of these concepts and the more general Hamburger moment problem see the monograph by Akhiezer [1].

In [8] the authors introduced a non-linear multiplicative transformation from Hausdorff moment sequences to Stieltjes moment sequences. In [9] we introduced a non-linear transformation $T$ of the set of Hausdorff moment sequences into itself by the formula:

$$
T\left(\left(a_{n}\right)\right)_{n}=1 /\left(a_{0}+a_{1}+\cdots+a_{n}\right), \quad n \geq 0 .
$$

* This work was initiated during the visit of the second author to the University of Copenhagen partially supported by D.G.E.S, ref. BFM2003-6335-C03-01, FQM-262 (Junta de Andalucía), and by grant 21-03-0324 from the Danish Natural Science Research Council.

Received November 14, 2006. 
The corresponding transformation of positive measures on $[0,1]$ is denoted $\widehat{T}$. We recall from [9] that if $v \neq 0$, then $\widehat{T}(v)(\{0\})=0$ and

$$
\int_{0}^{1} \frac{1-t^{z+1}}{1-t} d v(t) \int_{0}^{1} t^{z} d \widehat{T}(v)(t)=1 \quad \text { for } \quad \operatorname{Re} z \geq 0 .
$$

Assuming $\operatorname{Re} z>0$ we can consider $t^{z}=\exp (z \log t)$ as a continuous function on $[0,1]$ with value 0 for $t=0$. Likewise $\left(1-t^{z}\right) /(1-t)$ is a continuous function for $t \in[0,1]$ with value $z$ for $t=1$. If $\operatorname{Re} z=0, z \neq 0$ the function $t^{z}$ is only considered for $t>0$, so it is important that $\widehat{T}(v)$ has no mass at zero. Finally $t^{0} \equiv 1$. It is clear that if $v$ is a probability measure, then so is $\widehat{T}(v)$, and in this way we get a transformation of the convex set of normalized Hausdorff moment sequences (i.e. $a_{0}=1$ ) as well as a transformation of the set of probability measures on $[0,1]$. By Kakutani's theorem the transformation has a fixed point, and by (1.1) it is clear that a fixed point $\left(m_{n}\right)_{n}$ is uniquely determined by the recursive equation

$$
m_{0}=1, \quad\left(1+m_{1}+\cdots+m_{n}\right) m_{n}=1, \quad n \geq 1 .
$$

Therefore

$$
m_{n+1}^{2}+\frac{m_{n+1}}{m_{n}}-1=0
$$

giving

$$
m_{1}=\frac{-1+\sqrt{5}}{2}, \quad m_{2}=\frac{\sqrt{22+2 \sqrt{5}}-\sqrt{5}-1}{4}, \quad \ldots .
$$

The purpose of this paper is to study the Hausdorff moment sequence $\left(m_{n}\right)_{n}$ and to determine its associated probability measure $\mu$, called the fixed point measure.

We already know that $\mu(\{0\})=0$ because $\mu=\widehat{T}(\mu)$, but it is also convenient to notice that $\mu(\{1\})=0$. It is clear that $\left(m_{n}\right)_{n}$ decreases to $c=\mu(\{1\}) \geq 0$, hence $m_{0}+m_{1}+\cdots+m_{n} \geq(n+1) m_{n}$. By (1.3) we get $1 \geq(n+1) m_{n}^{2} \geq(n+1) c^{2}$, showing that $c=0$.

In Section 4 we prove much more, namely

$$
m_{n} \sim 1 / \sqrt{2 n} \quad \text { for } n \rightarrow \infty .
$$

We will study $\mu$ by determining what we call the Bernstein transform

$$
f(z)=\mathscr{B}(\mu)(z)=\int_{0}^{1} \frac{1-t^{z}}{1-t} d \mu(t), \quad \operatorname{Re} z>0
$$


as well as the Mellin transform

$$
F(z)=\mathscr{M}(\mu)(z)=\int_{0}^{1} t^{z} d \mu(t), \quad \operatorname{Re} z>0 .
$$

These functions are clearly holomorphic in the half-plane $\operatorname{Re} z>0$ and continuous in $\operatorname{Re} z \geq 0$, the latter because $\mu(\{0\})=0$.

As a first result we prove:

THEOREM 1.1. The functions $f, F$ can be extended to meromorphic functions in $\mathrm{C}$ and they satisfy

$$
\begin{gathered}
f(z+1) F(z)=1, \quad z \in \mathrm{C} \\
f(z)=f(z+1)-\frac{1}{f(z+1)}, \quad z \in \mathrm{C} .
\end{gathered}
$$

They are holomorphic in $\operatorname{Re} z>-1$. Furthermore $z=-1$ is a pole of $f$ and F.

The fixed point measure $\mu$ has the properties

$$
\int_{0}^{1} t^{x} d \mu(t)<\infty, \quad x>-1 ; \quad \int_{0}^{1} \frac{d \mu(t)}{t}=\infty .
$$

Proof. By (1.2) with $v$ replaced by the fixed point measure $\mu$ we get $f(z+1) F(z)=1$ for $\operatorname{Re} z \geq 0$, showing in particular that $f(z+1)$ and $F(z)$ are different from zero for $\operatorname{Re} z \geq 0$. For $\operatorname{Re} z \geq 0$ we get by (1.6)

$f(z+1)-f(z)=\int_{0}^{1} \frac{t^{z}-t^{z+1}}{1-t} d \mu(t)=\int_{0}^{1} t^{z} d \mu(t)=F(z)=\frac{1}{f(z+1)}$,

which shows (1.9) for these values of $z$.

We remark that $\operatorname{Re} f(z)>0$ and in particular $f(z) \neq 0$ for $\operatorname{Re} z>0$. This follows by (1.6) because $\operatorname{Re}\left(t^{z}\right) \leq\left|t^{z}\right|<1$ for $0<t<1$ and $\operatorname{Re} z>0$.

We next use equation (1.9) to define $f(z)$ for $\operatorname{Re} z \geq-1$, yielding a holomorphic continuation of $f$ to the open half-plane $\operatorname{Re} z>-1$ because $f(z+1) \neq 0$.

Using equation (1.9) once more we obtain a meromorphic extension of $f$ to the half-plane $\operatorname{Re} z>-2$. There will be poles at points $z$ for which $f(z+1)=0$, in particular for $z=-1$ because $f(0)=0$.

Repeated use of equation (1.9) makes it possible to obtain a meromorphic extension to $C$. At each step, $z$ will be a pole if $z+1$ is a zero or a pole. 
At this stage we cannot give a complete picture of the poles of $f$, but we return to that in Theorem 1.4.

Having extended $f$ to a meromorphic function in $C$ such that (1.9) holds, we extend $F$ to a meromorphic function in $C$ such that equation (1.8) holds.

Let us notice that also $F$ has no poles in $\operatorname{Re} z>-1$ because $f(z+1) \neq 0$. Moreover $z=-1$ is a pole of $F$ because $f(0)=0$.

By a classical result (going back to Landau for Dirichlet series), see [23, p. 58], we then get equation (1.10).

The function $f$ can be characterized in analogy with the Bohr-Mollerup theorem about the Gamma function, cf. [2]. More precisely we have:

THEOREM 1.2. The Bernstein transform (1.6) of the fixed point measure is a function $f:] 0, \infty[\rightarrow] 0, \infty[$ with the following properties

(i) $f(1)=1$,

(ii) $\log (1 / f)$ is convex,

(iii) $f(s)=f(s+1)-1 / f(s+1), s>0$.

Conversely, if $\tilde{f}:] 0, \infty[\rightarrow] 0, \infty[$ satisfies (i)-(iii), then it is equal to $f$ and for $0<s \leq 1$ we have

$$
\tilde{f}(s)=\lim _{n \rightarrow \infty} \psi^{\circ n}\left(\frac{1}{m_{n-1}}\left(\frac{m_{n-1}}{m_{n}}\right)^{s}\right),
$$

where $\psi$ is the rational function $\psi(z)=z-1 / z$. In particular (1.11) holds for $f$.

Here and elsewhere we use the notation for composition of mappings $\psi^{\circ 1}(z)=\psi(z), \psi^{\circ n}(z)=\psi\left(\psi^{\circ(n-1)}(z)\right), n \geq 2$. Theorem 1.2 will be proved in Section 3. Using the relation $f(s+1) F(s)=1$ it is clear that Theorem 1.2 can be reformulated to a characterization of $F$ :

THeOREM 1.3. There exists one and only one function $F:]-1, \infty[\rightarrow$ ] $0, \infty[$ with the following properties

(i) $F(0)=1$,

(ii) $F$ is log-convex,

(iii) $1 / F(s)=1 / F(s+1)-F(s+1), s>-1$,

namely $F$ is the Mellin transform

$$
F(s)=\int_{0}^{1} t^{s} d \mu(t), \quad s>-1
$$

of the fixed point measure. 
Let $\mathscr{H}$ denote the set of normalized Hausdorff moment sequences considered as a subset of $[0,1]^{\mathrm{N}_{0}}$ with the product topology, $\mathrm{N}_{0}=\{0,1, \ldots\}$. In Section 2 we prove that the fixed point $\mathbf{m}=\left(m_{n}\right)_{n}$ is attractive in the sense that for each $\mathbf{a}=\left(a_{n}\right)_{n} \in \mathscr{H}$ the sequence of iterates $T^{\circ n}(\mathbf{a})$ converges to $\mathbf{m}$ in $\mathscr{H}$. Focusing on probability measures we see that every probability measure $\tau$ on $[0,1]$ belongs to the domain of attraction of the fixed point measure $\mu$ in the sense that $\lim _{n \rightarrow \infty} \widehat{T}^{\circ n}(\tau)=\mu$ weakly. For $q \in \mathbf{R}$ we denote by $\delta_{q}$ the probability measure with mass 1 concentrated at the point $q$. By specializing the iteration using $\tau=\delta_{0}$ we prove the following result:

THEOREM 1.4. Let $f$ and $F$ be the meromorphic functions in $C$ extending (1.6) and (1.7) respectively. The zeros and poles of $f$ are all simple and are contained in $]-\infty, 0]$. The zeros of $f$ are denoted $\xi_{0}=0$ and $\xi_{p, k}, p=$ $1,2, \ldots, k=1, \ldots, 2^{p-1}$ with $-p-1<\xi_{p, 1}<\xi_{p, 2}<\cdots<\xi_{p, 2^{p-1}}<-p$.

The poles of $f$ are $-l, \xi_{p, k}-l, l=1,2, \ldots$ with $p, k$ as above.

Defining

$$
\rho_{0}=\frac{1}{f^{\prime}(0)} ; \quad \rho_{p, k}=\frac{1}{f^{\prime}\left(\xi_{p, k}\right)},
$$

then $\rho_{0}, \rho_{p, k}>0$.

The following representations hold

$$
F(z)=\frac{\rho_{0}}{z+1}+\sum_{p=1}^{\infty} \sum_{k=1}^{2^{p-1}} \frac{\rho_{p, k}}{z+1-\xi_{p, k}},
$$

and

$$
f(z)=z \sum_{l=1}^{\infty}\left[\frac{\rho_{0}}{l(z+l)}+\sum_{p=1}^{\infty} \sum_{k=1}^{2^{p-1}} \frac{\rho_{p, k}}{\left(l-\xi_{p, k}\right)\left(z+l-\xi_{p, k}\right)}\right] .
$$

The fixed point measure $\mu$ has an increasing and convex density $\mathscr{D}$ with respect to Lebesgue measure on ]0, 1[ and it is given by

$$
\mathscr{D}(t)=\rho_{0}+\sum_{p=1}^{\infty} \sum_{k=1}^{2^{p-1}} \rho_{p, k} t^{-\xi_{p, k}} .
$$

While clearly $\mathscr{D}(0)=\rho_{0}$, we prove in Theorem 3.9 that

$$
\mathscr{D}(t) \sim 1 / \sqrt{2 \pi(1-t)}, \quad t \rightarrow 1 .
$$


It is possible to obtain expressions for $\xi_{p, k}$ and $\rho_{p, k}$ in terms of the moments $\left(m_{n}\right)$. This is quite technical and is given in Theorem 3.8.

We recall that a function $\varphi$ is called a Stieltjes transform if it can be written in the form

$$
\left.\left.\varphi(z)=a+\int_{0}^{\infty} \frac{d \sigma(x)}{x+z}, \quad z \in \mathrm{C} \backslash\right]-\infty, 0\right],
$$

where $a \geq 0$ and $\sigma$ is a positive measure on [0, $\infty$ [ such that (1.16) makes sense, i.e. $\int 1 /(x+1) d \sigma(x)<\infty$.

It is clear that if $\sigma \neq 0$ then $\varphi$ is strictly decreasing on $] 0, \infty[$ with $a=$ $\lim _{s \rightarrow \infty} \varphi(s)$. Furthermore, $\varphi$ is holomorphic in $\left.\left.C \backslash\right]-\infty, 0\right]$ with

$$
\frac{\operatorname{Im} \varphi(z)}{\operatorname{Im} z}<0 \quad \text { for } \quad z \in \mathrm{C} \backslash \mathbf{R},
$$

so in particular $\varphi$ is never zero in $C \backslash]-\infty, 0]$. The Stieltjes transforms we are going to consider will be meromorphic in $\mathrm{C}$. The function (1.16) is meromorphic precisely when the measure $\sigma$ is discrete and the set of mass-points have no finite accumulation points, i.e. if and only if

$$
\varphi(z)=a+\sum_{p=0}^{\infty} \frac{\sigma_{p}}{z+\eta_{p}}
$$

with $\sigma_{p}>0,0 \leq \eta_{0}<\eta_{1}<\eta_{2}<\cdots \rightarrow \infty$.

For results about Stieltjes transforms see [10]. Stieltjes transforms are closely related to Pick functions, cf. [1], [16]. We recall that a Pick function is a holomorphic function $\varphi: C \backslash R \rightarrow C$ satisfying

$$
\frac{\operatorname{Im} \varphi(z)}{\operatorname{Im} z} \geq 0 \quad \text { for } \quad z \in \mathrm{C} \backslash \mathbf{R},
$$

so if $\varphi \neq 0$ is a Stieltjes transform, then $1 / \varphi$ is a Pick function. Notice that $z /(z+a)$ is a Pick function for any $a>0$.

Corollary 1.5. In the notation of Theorem $1.4 f(z) / z$ and $F(z)$ are Stieltjes transforms and $f$ is a Pick function.

We have used the name Bernstein transform for (1.6). In general, if $v$ is a positive finite measure on $] 0,1]$, we call

$$
\mathscr{B}(v)(z)=\int_{0}^{1} \frac{1-t^{z}}{1-t} d v(t)
$$


the Bernstein transform of $v$, because it is a Bernstein function in the terminology of [10]. In fact we can write

$$
\mathscr{B}(v)(z)=v(\{1\}) z+\int_{0}^{\infty}\left(1-e^{-x z}\right) d \lambda(x), \quad \operatorname{Re} z \geq 0,
$$

where $\lambda$ is defined as the image measure of $(1-t)^{-1}(v \mid] 0,1[)$ under $\log (1 / x)$ mapping ]0, 1 [ onto ]0, $\infty[$. We recall that $\lambda$ is called the Lévy measure of the Bernstein function. It follows that $\mathscr{B}(v)^{\prime}$ is a completely monotonic function. Bernstein functions are very important in the theory of Lévy processes, see [11].

In Section 4 we prove that $\left(m_{n}\right)_{n}$ is infinitely divisible in the sense that $\left(m_{n}^{\alpha}\right)_{n}$ is a Hausdorff moment sequence for all $\alpha>0$.

\section{An iteration leading to the fixed point measure}

For $n=0,1, \ldots$ we denote the moments of $\mu_{n}=\widehat{T}^{\circ n}\left(\delta_{0}\right)$ by $\left(m_{n, k}\right)_{k}$, i.e.

$$
\int_{0}^{1} t^{k} d \widehat{T}^{o n}\left(\delta_{0}\right)(t)=m_{n, k}
$$

hence for $n \geq 1$

$$
m_{n, k}=\left(m_{n-1,0}+m_{n-1,1}+\cdots+m_{n-1, k}\right)^{-1} .
$$

Notice that $m_{n, 0}=1$ for all $n$ and $m_{0, k}=\delta_{0 k}, m_{1, k}=1, m_{2, k}=1 /(k+1)$ for all $k$.

LEMma 2.1. For fixed $k=0,1, \ldots$ we have

$$
\begin{aligned}
& m_{0, k} \leq m_{2, k} \leq m_{4, k} \leq \cdots \\
& m_{1, k} \geq m_{3, k} \geq m_{5, k} \geq \cdots
\end{aligned}
$$

and these sequences have the same limit

$$
\lim _{n \rightarrow \infty} m_{2 n, k}=\lim _{n \rightarrow \infty} m_{2 n+1, k}=m_{k}
$$

where $\left(m_{k}\right)_{k}$ is the fixed point given by (1.3).

Furthermore, $\lim _{k \rightarrow \infty} m_{n, k}=0$ for $n \geq 2$, implying that $\mu_{n}=\widehat{T}^{\circ n}\left(\delta_{0}\right)$ has no mass at $t=1$ for $n \geq 2$.

Proof. Since the result is trivial for $k=0$, we assume that $k \geq 1$ and have

$$
0=m_{0, k}<m_{2, k}=\frac{1}{k+1} ; \quad 1=m_{1, k}>m_{3, k}=\frac{1}{\mathscr{H}_{k+1}},
$$


where $\mathscr{H}_{p}=1+\frac{1}{2}+\cdots+\frac{1}{p}$ is the $p^{\prime}$ th harmonic number. We now get

$$
\frac{1}{m_{4, k}}=\sum_{j=0}^{k} m_{3, j}<k+1
$$

hence $m_{4, k}>m_{2, k}$. We next use this to conclude

$$
\frac{1}{m_{5, k}}=\sum_{j=0}^{k} m_{4, j}>\sum_{j=0}^{k} m_{2, j}=\frac{1}{m_{3, k}},
$$

hence $m_{5, k}<m_{3, k}$. It is clear that this procedure can be continued and reformulated to a proof by induction.

Defining

$$
m_{k}^{\prime}=\lim _{n \rightarrow \infty} m_{2 n, k}, \quad m_{k}^{\prime \prime}=\lim _{n \rightarrow \infty} m_{2 n+1, k},
$$

we get the following relations from (2.1)

$$
m_{k}^{\prime}=\left(1+m_{1}^{\prime \prime}+\cdots+m_{k}^{\prime \prime}\right)^{-1}, \quad m_{k}^{\prime \prime}=\left(1+m_{1}^{\prime}+\cdots+m_{k}^{\prime}\right)^{-1}, \quad k \geq 1,
$$

because clearly $m_{0}^{\prime}=m_{0}^{\prime \prime}=m_{0}=1$. It follows easily by induction using (2.2) that $m_{k}^{\prime}=m_{k}^{\prime \prime}=m_{k}$ for all $k$.

Since $m_{2 n, k} \leq m_{k}$ we get $\lim _{k \rightarrow \infty} m_{2 n, k}=0$. Furthermore, for $n \geq 1$

$$
\frac{1}{m_{2 n+1, k}}=\sum_{j=0}^{k} m_{2 n, j} \geq \sum_{j=0}^{k} m_{2, j}=\mathscr{H}_{k+1}
$$

and hence $\lim _{k \rightarrow \infty} m_{2 n+1, k}=0$.

We recall that $\mathscr{H}$ denotes the set of normalized Hausdorff moment sequences $\mathbf{a}=\left(a_{n}\right)_{n}$. The mapping $v \rightarrow\left(\int x^{n} d v(x)\right)_{n}$ from the set of probability measures $v$ on $[0,1]$ to $\mathscr{H}$ is a homeomorphism between compact sets, when the set of probability measures carries the weak topology and $\mathscr{H}$ carries the topology inherited from $[0,1]^{\mathrm{N}_{0}}$ equipped with the product topology.

Defining an order relation $\leq$ on $\mathscr{H}$ by writing $\mathbf{a} \leq \mathbf{b}$ if $a_{k} \leq b_{k}$ for $k=$ $0,1, \ldots$, we easily get the following Lemma:

Lemma 2.2. The transformation $T: \mathscr{H} \rightarrow \mathscr{H}$ is decreasing, i.e.

$$
\mathbf{a} \leq \mathbf{b} \Rightarrow T(\mathbf{a}) \geq T(\mathbf{b}) .
$$

Theorem 2.3. For every $\mathbf{a} \in \mathscr{H}$ we have

$$
\lim _{n \rightarrow \infty} T^{\circ n}(\mathbf{a})=\mathbf{m}
$$


where $\mathbf{m}=\left(m_{n}\right)_{n}$ is the fixed point.

Proof. For $0 \leq q \leq 1$ we write $\underline{\boldsymbol{q}}=\left(q^{n}\right)_{n}$, hence $\underline{\mathbf{0}} \leq \mathbf{a} \leq \underline{\mathbf{1}}$ for every $\mathbf{a} \in \mathscr{H}$. By Lemma 2.2 we get

$$
\begin{array}{r}
T^{\circ(2 n)}(\underline{\mathbf{0}}) \leq T^{\circ(2 n)}(\mathbf{a}) \leq T^{\circ(2 n)}(\underline{\mathbf{1}})=T^{\circ(2 n+1)}(\underline{\mathbf{0}}) \\
T^{\circ(2 n+1)}(\underline{\mathbf{0}}) \geq T^{\circ(2 n+1)}(\mathbf{a}) \geq T^{\circ(2 n+1)}(\underline{\mathbf{1}})=T^{\circ(2 n+2)}(\underline{\mathbf{0}}),
\end{array}
$$

and since $\lim _{n \rightarrow \infty} T^{\circ n}(\underline{\mathbf{0}})=\mathbf{m}$ by Lemma 2.1, we get

$$
\lim _{n \rightarrow \infty} T^{\circ(2 n)}(\mathbf{a})=\lim _{n \rightarrow \infty} T^{\circ(2 n+1)}(\mathbf{a})=\mathbf{m} .
$$

Theorem 2.3 can also be expressed that $\widehat{T}^{\circ n}(\tau) \rightarrow \mu$ weakly for any probability measure $\tau$ on $[0,1]$. Specializing this to $\tau=\delta_{0}$ and using formula (1.2), we obtain:

COROLlaRY 2.4. The iterated sequence $\mu_{n}=\widehat{T}^{\circ n}\left(\delta_{0}\right)$ of measures converges weakly to the fixed point measure $\mu$ and

$$
\int_{0}^{1} \frac{1-t^{z+1}}{1-t} d \mu_{n}(t) \int_{0}^{1} t^{z} d \mu_{n+1}(t)=1, \quad \operatorname{Re} z \geq 0, n=0,1, \ldots
$$

We have $\mu_{0}=\delta_{0}, \mu_{1}=\delta_{1}, \mu_{2}=\chi_{] 0,1[}(t) d t$, where $\chi_{10,1[}(t)$ denotes the indicator function for the interval ]0, 1[. The Bernstein transform of the measure $\mu_{2}$ is

$$
\mathscr{B}\left(\mu_{2}\right)(z)=\int_{0}^{1} \frac{1-t^{z}}{1-t} d t=\sum_{l=1}^{\infty}\left(\frac{1}{l}-\frac{1}{z+l}\right)=\Psi(z+1)+\gamma,
$$

where $\gamma$ is Euler's constant and $\Psi(x)=\Gamma^{\prime}(x) / \Gamma(x)$ is the Digamma function.

The measure $\mu_{3}$ has been calculated in [9] and the result is

$$
\mu_{3}=\left(\sum_{p=0}^{\infty} \alpha_{p} t^{-\xi_{p}}\right) \chi_{] 0,1[}(t) d t
$$

where $0=\xi_{0}>\xi_{1}>\xi_{2}>\cdots$ satisfy $-p-1<\xi_{p}<-p$ for $p=$ $1,2, \ldots$ and $\alpha_{p}>0, p=0,1, \ldots$ More precisely, it was proved that $\xi_{p}$ is the unique solution $x \in]-p-1,-p$ [ of the equation $\Psi(1+x)=-\gamma$. Writing $\xi_{p}=-p-1+\delta_{p}$, we have $0<\delta_{p+1}<\delta_{p}<\frac{1}{2}, \delta_{p} \sim 1 / \log p, p \rightarrow \infty$. Furthermore, $\alpha_{p}=1 / \Psi^{\prime}\left(1+\xi_{p}\right) \sim 1 / \log ^{2} p$. Since $\sum \alpha_{p} /\left(1-\xi_{p}\right)=1$, we have the crude estimate $\alpha_{p}<p+2$. 
We shall now prove that all the measures $\mu_{n}, n \geq 4$ have a form similar to that of $\mu_{3}$.

LEMмA 2.5. For $n \geq 3$ the measure $\mu_{n}$ has the form

$$
\mu_{n}=\left(\rho_{0}^{(n)}+\sum_{p=1}^{\infty} \sum_{k=1}^{N(n, p)} \rho_{p, k}^{(n)} t^{-\xi_{p, k}^{(n)}}\right) \chi_{] 0,1[}(t) d t
$$

where for each $p \geq 1$

(i) $1 \leq N(n, p) \leq 2^{p-1}$,

(ii) $-p-1<\xi_{p, 1}^{(n)}<\xi_{p, 2}^{(n)}<\cdots<\xi_{p, N(n, p)}^{(n)}<-p$,

(iii) $0<\rho_{0}^{(n)}<1,0<\rho_{p, k}^{(n)}<p+2, k=1, \ldots, N(n, p)$.

Proof. The result for $n=3$ follows from the description above from [9] with $\rho_{0}^{(3)}=\alpha_{0}, N(3, p)=1, \rho_{p, 1}^{(3)}=\alpha_{p}, \xi_{p, 1}^{(3)}=\xi_{p}$.

Assume now that the result holds for $\mu_{n}$ and let us prove it for $\mu_{n+1}$. For $\operatorname{Re} z>0$ we then have

$$
\begin{aligned}
f_{n}(z) & :=\mathscr{B}\left(\mu_{n}\right)(z) \\
= & \int_{0}^{1} \frac{1-t^{z}}{1-t} d \mu_{n}(t)=\sum_{l=0}^{\infty} \int_{0}^{1}\left(t^{l}-t^{z+l}\right) d \mu_{n}(t) \\
& =\sum_{l=0}^{\infty}\left[\rho_{0}^{(n)} \int_{0}^{1}\left(t^{l}-t^{z+l}\right) d t+\sum_{p=1}^{\infty} \sum_{k=1}^{N(n, p)} \rho_{p, k}^{(n)} \int_{0}^{1}\left(t^{l-\xi_{p, k}^{(n)}}-t^{z+l-\xi_{p, k}^{(n)}}\right) d t\right] \\
& =z \sum_{l=1}^{\infty}\left[\frac{\rho_{0}^{(n)}}{l(z+l)}+\sum_{p=1}^{\infty} \sum_{k=1}^{N(n, p)} \frac{\rho_{p, k}^{(n)}}{\left(l-\xi_{p, k}^{(n)}\right)\left(z+l-\xi_{p, k}^{(n)}\right)}\right] .
\end{aligned}
$$

This shows that $f_{n}(z) / z$ is a Stieltjes transform and a meromorphic function in $\mathrm{C}$ with poles at the points

$$
-l, \xi_{p, k}^{(n)}-l, \quad l=1,2, \ldots, \quad p=1,2, \ldots, \quad k=1, \ldots, N(n, p),
$$

so in the interval $]-p-1,-p]$ we have the poles

$$
-p, \xi_{p-l, k}^{(n)}-l, \quad k=1, \ldots, N(n, p-l), l=1, \ldots, p-1 .
$$

Since $f_{n}(x) / x$ is strictly decreasing between the poles, we conclude that there is precisely one simple zero between two consecutive poles. Let $N(n+1, p)$ denote the number of zeros of $f_{n}$ in $]-p-1,-p$ [ and let $\xi_{p, k}^{(n+1)}$ denote the zeros numbered such that

$$
-p-1<\xi_{p, 1}^{(n+1)}<\xi_{p, 2}^{(n+1)}<\cdots<\xi_{p, N(n+1, p)}^{(n+1)}<-p .
$$


In addition also $z=0$ is a zero of $f_{n}$. There are no zeros or poles in $\left.\left.C \backslash\right]-\infty, 0\right]$ because $f_{n}(z) / z$ is a Stieltjes transform.

We are now ready to prove equation (2.5) and (i)-(iii) with $n$ replaced by $n+1$.

(i). By (2.6) we get

$$
N(n+1, p) \leq 1+\sum_{l=1}^{p-1} N(n, p-l) \leq 1+\sum_{l=1}^{p-1} 2^{p-l-1}=2^{p-1} .
$$

(ii) is clear by definition, when we have proved that the measure $\mu_{n+1}$ has the form (2.5) using the numbers $\xi_{p, k}^{(n+1)}$.

(iii). By a classical result, see [19], [18], [4], $1 / f_{n}(z)$ is a Stieltjes transform because $f_{n}(z) / z$ is so, i.e.

$$
\frac{1}{f_{n}(z)}=\frac{\rho_{0}^{(n+1)}}{z}+\sum_{p=1}^{\infty} \sum_{k=1}^{N(n+1, p)} \frac{\rho_{p, k}^{(n+1)}}{z-\xi_{p, k}^{(n+1)}},
$$

with $\rho_{0}^{(n+1)}, \rho_{p, k}^{(n+1)}>0$. There is no constant term in the Stieltjes representation because $f_{n}(x) \rightarrow \infty$ for $x \rightarrow \infty$. In fact, by Lemma 2.1 we get

$$
\lim _{x \rightarrow \infty} f_{n}(x)=\int_{0}^{1} \frac{d \mu_{n}(t)}{1-t}=\sum_{k=0}^{\infty} m_{n, k}=\lim _{k \rightarrow \infty} \frac{1}{m_{n+1, k}}=\infty .
$$

Note that

$$
\rho_{0}^{(n+1)}=\frac{1}{f_{n}^{\prime}(0)}, \quad \rho_{p, k}^{(n+1)}=\frac{1}{f_{n}^{\prime}\left(\xi_{p, k}^{(n+1)}\right)} .
$$

By (2.3) we get

$$
\int_{0}^{1} t^{z} d \mu_{n+1}(t)=\frac{1}{f_{n}(z+1)}=\frac{\rho_{0}^{(n+1)}}{z+1}+\sum_{p=1}^{\infty} \sum_{k=1}^{N(n+1, p)} \frac{\rho_{p, k}^{(n+1)}}{z+1-\xi_{p, k}^{(n+1)}}
$$

which shows that

$$
\mu_{n+1}=\left(\rho_{0}^{(n+1)}+\sum_{p=1}^{\infty} \sum_{k=1}^{N(n+1, p)} \rho_{p, k}^{(n+1)} t^{-\xi_{p, k}^{(n+1)}}\right) \chi_{] 0,1[}(t) d t
$$

which is (2.5) with $n$ replaced by $n+1$. 
Since $\mu_{n+1}$ is a probability measure we get

$$
\rho_{0}^{(n+1)}<1, \quad \rho_{p, k}^{(n+1)} \int_{0}^{1} t^{-\xi_{p, k}^{(n+1)}} d t<1,
$$

hence

$$
\rho_{p, k}^{(n+1)}<1-\xi_{p, k}^{(n+1)}<p+2
$$

Corollary 2.6. For $n \geq 0$ let $\mu_{n}=\widehat{T}^{o n}\left(\delta_{0}\right)$. The functions $f_{n}=\mathscr{B}\left(\mu_{n}\right)$ are meromorphic Pick functions and the functions $F_{n}=\mathscr{M}\left(\mu_{n}\right)$ are meromorphic Stielties transforms satisfying

$$
f_{n}(z+1) F_{n+1}(z)=1, \quad z \in \mathrm{C} .
$$

All zeros and poles of $f_{n}$ are contained in $\left.]-\infty, 0\right]$.

Proof. We have $f_{0}(z)=1, f_{1}(z)=z, F_{0}(z)=0, F_{1}(z)=1, F_{2}(z)=$ $1 /(z+1)$ and for $n \geq 2$ the result follows from Lemma 2.5 and its proof.

In order to obtain a limit result for $n \rightarrow \infty$ in Corollary 2.6 we need the following:

LeMma 2.7. Let $\left(\varphi_{n}\right)_{n}$ be a sequence of Stieltjes transforms of the form

$$
\varphi_{n}(z)=\int_{0}^{\infty} \frac{d \sigma_{n}(x)}{x+z}, \quad n=1,2, \ldots
$$

and assume that $\varphi_{n}(z) \rightarrow \varphi(z)$ uniformly on compact subsets of $\operatorname{Re} z>0$ for some holomorphic function $\varphi$ on the right half-plane.

Then $\varphi$ is a Stieltjes transform

$$
\varphi(z)=a+\int_{0}^{\infty} \frac{d \sigma(x)}{x+z}
$$

and $\lim _{n \rightarrow \infty} \sigma_{n}=\sigma$ vaguely. Furthermore, $\varphi_{n}(z) \rightarrow \varphi(z)$ uniformly on compact subsets of $\mathrm{C} \backslash]-\infty, 0]$.

Proof. Since

$$
\int_{0}^{\infty} \frac{d \sigma_{n}(x)}{x+1}=\varphi_{n}(1) \rightarrow \varphi(1),
$$

there exists a constant $K>0$ such that $\int 1 /(x+1) d \sigma_{n}(x) \leq K$ for all $n$. Let $\sigma$ be a vague accumulation point for $\left(\sigma_{n}\right)_{n}$. Replacing $\left(\sigma_{n}\right)_{n}$ by a subsequence we can assume without loss of generality that $\sigma_{n} \rightarrow \sigma$ vaguely. By standard results in measure theory, cf. [7, Prop. 4.4], we have

$$
\int_{0}^{\infty} \frac{d \sigma(x)}{x+1} \leq K, \quad \lim _{n \rightarrow \infty} \int f d \sigma_{n}=\int f d \sigma
$$


for any continuous function $f:[0, \infty[\rightarrow \mathrm{C}$ which is $o(1 /(x+1))$ for $x \rightarrow \infty$. In particular

$$
\left.\left.\varphi_{n}^{\prime}(z)=-\int_{0}^{\infty} \frac{d \sigma_{n}(x)}{(x+z)^{2}} \rightarrow-\int_{0}^{\infty} \frac{d \sigma(x)}{(x+z)^{2}}, \quad z \in \mathrm{C} \backslash\right]-\infty, 0\right],
$$

showing that

$$
\varphi^{\prime}(z)=-\int_{0}^{\infty} \frac{d \sigma(x)}{(x+z)^{2}}, \quad \operatorname{Re} z>0
$$

hence

$$
\varphi(z)=a+\int_{0}^{\infty} \frac{d \sigma(x)}{x+z}, \quad \operatorname{Re} z>0
$$

for some constant $a$. Using $\varphi(x)=\lim _{n \rightarrow \infty} \varphi_{n}(x) \geq 0$ for $x>0$, we get $a \geq 0$, showing that $\varphi$ is a Stieltjes transform. By uniqueness of $a$ and $\sigma$ in the representation of $\varphi$ as a Stieltjes transform, we conclude that the accumulation point $\sigma$ is unique, hence $\lim _{n \rightarrow \infty} \sigma_{n}=\sigma$ vaguely.

It is now easy to see that $\left(\varphi_{n}(z)\right)_{n}$ is uniformly bounded on compact subsets of $C \backslash]-\infty, 0]$, and the last assertion of Lemma 2.7 is a consequence of the Stieltjes-Vitali theorem.

Proof of Theorem 1.4. From Lemma 2.5 follows that the Mellin transform $\mathcal{M}\left(\mu_{n}\right)(z)$ coincides on $\operatorname{Re} z \geq 0$ with the meromorphic function

$$
\frac{\rho_{0}^{(n)}}{z+1}+\sum_{p=1}^{\infty} \sum_{k=1}^{N(n, p)} \frac{\rho_{p, k}^{(n)}}{z+1-\xi_{p, k}^{(n)}}=\int_{0}^{\infty} \frac{d \sigma_{n}(x)}{x+z},
$$

where $\sigma_{n}$ is the discrete measure

$$
\sigma_{n}=\rho_{0}^{(n)} \delta_{1}+\sum_{p=1}^{\infty} \sum_{k=1}^{N(n, p)} \rho_{p, k}^{(n)} \delta_{1-\xi_{p, k}^{(n)}}
$$

Since $\mathcal{M}\left(\mu_{n}\right)(z) \rightarrow \mathcal{M}(\mu)(z)$ uniformly on compact subsets of $\operatorname{Re} z>0$ by Corollary 2.4 , it follows by Lemma 2.7 that $\mathscr{M}(\mu)$ is a Stieltjes transform

$$
\mathscr{M}(\mu)(z)=a+\int_{0}^{\infty} \frac{d \sigma(x)}{x+z},
$$

and $\sigma_{n} \rightarrow \sigma$ vaguely. Since $\mathcal{M}(\mu)(k)=m_{k} \rightarrow 0$ as $k \rightarrow \infty$, we get $a=0$. Using that $\sigma_{n}$ has at most $2^{p-1}$ mass points in $[p+1, p+2], p=1,2, \ldots$ and that $\rho_{p, k}^{(n)}<p+2$ by Lemma 2.5 , we can write

$$
\sigma=\rho_{0} \delta_{1}+\sum_{p=1}^{\infty} \sum_{k=1}^{N_{p}} \rho_{p, k} \delta_{1-\xi_{p, k}},
$$


with $\rho_{0} \geq 0,0<\rho_{p, k} \leq p+2$ and $-p-1 \leq \xi_{p, 1}<\xi_{p, 2}<\cdots<\xi_{p, N_{p}}<-p$, where $N_{p} \leq 2^{p-1}$. At this stage we cannot confirm that $\rho_{0}>0,-p-1<\xi_{p, 1}$, $N_{p}=2^{p-1}$ and that $\xi_{p, k}$ are the zeros of $f$. The function

$$
\frac{\rho_{0}}{z+1}+\sum_{p=1}^{\infty} \sum_{k=1}^{N_{p}} \frac{\rho_{p, k}}{z+1-\xi_{p, k}}
$$

is a meromorphic extension of $\mathcal{M}(\mu)$ and therefore equal to the meromorphic function $F$ of Theorem 1.1. This shows that $\mu$ has the density

$$
\mathscr{D}(t)=\rho_{0}+\sum_{p=1}^{\infty} \sum_{k=1}^{N_{p}} \rho_{p, k} t^{-\xi_{p, k}},
$$

which is clearly increasing and convex since $-\xi_{p, k} \geq 1$. Finally, by (2.10) the Bernstein transform $\mathscr{B}(\mu)$ has the meromorphic extension

$$
z \sum_{l=1}^{\infty}\left[\frac{\rho_{0}}{l(z+l)}+\sum_{p=1}^{\infty} \sum_{k=1}^{N_{p}} \frac{\rho_{p, k}}{\left(l-\xi_{p, k}\right)\left(z+l-\xi_{p, k}\right)}\right],
$$

which is a Pick function. The function given by (2.11) equals the meromorphic function $f$ of Theorem 1.1. By Lemma 2.7 applied to the Stieltjes transforms $f_{n}(z) / z$, we conclude that $f_{n}(z) \rightarrow f(z)$ uniformly on compact subsets of $\mathrm{C} \backslash]-\infty, 0]$.

We already know from Theorem 1.1 that $F$ has a pole at $z=-1$ and hence $\rho_{0}>0$. The remaining poles of $F$ are $\xi_{p, k}-1$, so by formula (1.8) the zeros of $f$ are $z=0$ and $z=\xi_{p, k}$. By the expression (2.11) for $f$ the poles of $f$ are $-l, \xi_{p, k}-l$ and therefore $-p-1<\xi_{p, 1}, p=1,2, \ldots$.

We have now proved that the zeros and poles of $f$ are all simple and are contained in $]-\infty, 0]$. Since $f(z+1) F(z)=1$ we get by (2.9) that

$$
\frac{1}{f(z)}=\frac{\rho_{0}}{z}+\sum_{p=1}^{\infty} \sum_{k=1}^{N_{p}} \frac{\rho_{p, k}}{z-\xi_{p, k}}
$$

which shows equation (1.12).

To finish the proof we shall establish that $N_{p}=2^{p-1}$.

From the functional equation (1.9) and the fact that $f$ is strictly increasing between the poles, we see the following about the generation of zeros and poles of $f$ :

(1) If $z+1$ is regular point, then $f(z+1)= \pm 1$ if and only if $f(z)=0$. 
(2) If $z+1$ is regular point, then $f(z+1)=0$ if and only if $z$ is a pole. In the affirmative case $\operatorname{Res}(f, z)=-1 / f^{\prime}(z+1)$.

(3) If $z+1$ is a pole then $z$ is a pole with the same residue as in $z+1$.

(4) For a pole $\beta$ let $\alpha_{\beta}$ be the smallest zero in $] \beta, \infty\left[\right.$. Then $f(] \beta, \alpha_{\beta}[)=$ ]$-\infty, 0\left[\right.$ and there exists a unique point $x_{*}$ in $] \beta, \alpha_{\beta}$ [ such that $f\left(x_{*}\right)=$ -1 .

(5) For a pole $\beta$ let $\gamma_{\beta}$ be the biggest zero in $]-\infty, \beta\left[\right.$. Then $f(] \gamma_{\beta}, \beta[)=$ ] $0, \infty\left[\right.$ and there exists a unique point $x^{*}$ in $] \gamma_{\beta}, \beta$ [ such that $f\left(x^{*}\right)=1$.

From (1)-(5) we deduce that $f$ has the following properties. Since $f(0)=0$ we see that $f$ has poles at $z=-1,-2, \ldots$ in accordance with (2.11). There are no poles in ]-2, -1 [ since $f$ is regular in $]-1,0[$ and non-zero. Notice that $f$ is strictly increasing on $]-1, \infty$ [ mapping this interval onto the whole real line by (2.11). There is a unique point $\left.x_{*} \in\right]-1,0$ [ such that $f\left(x_{*}\right)=-1$, hence $x_{*}-1$ is a zero and $x_{*}-2, x_{*}-3, \ldots$ are poles. In $\left.]-3,-2\right]$ there are two poles namely $x_{*}-2$ and -2 and since $f$ is strictly increasing between consecutive poles we have two zeros in ] $-3,-2[$. By induction it is easy to see that there are exactly $2^{p-1}$ poles in each interval $\left.]-p-1,-p\right]$ and $2^{p-1}$ zeros in the open interval $]-p-1,-p\left[, p \geq 1\right.$. This shows that $N_{p}=2^{p-1}$. Note that $\xi_{1,1}=x_{*}-1$.

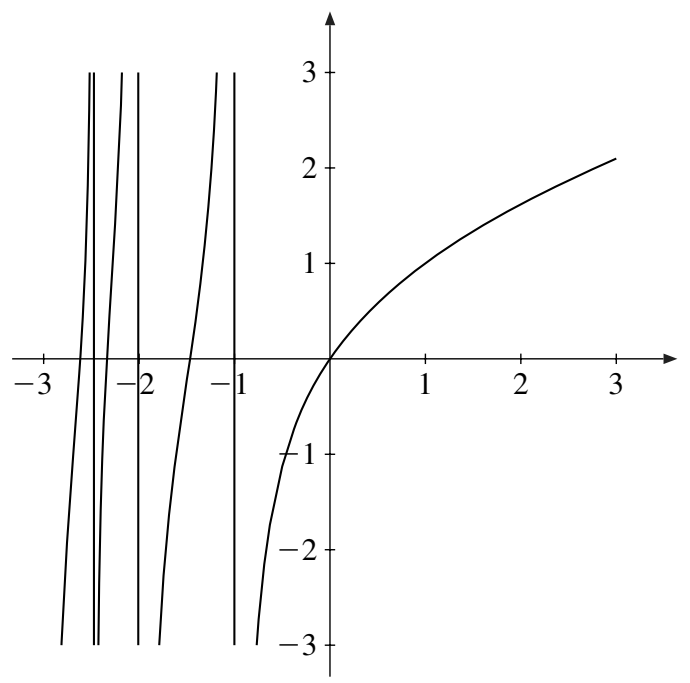

FIgURE 1. The graph of $f$ with vertical lines at the poles.

We give some further information about the poles of $f$.

We call the negative integers poles of the first generation of $f$ and say that a pole of $f$ is of the $l$-th generation, $l \geq 2$, if it is generated by a zero $\xi_{l-1, k}$, 
i.e. the pole is of the form $\xi_{l-1, k}-m$, for some integer $m \geq 1$. Then it can easily be proved by induction on $p$ that:

(1) In $]-p-1,-p$ ] there is one pole of the first generation (namely, $-p$ ), one pole of the second generation (namely $\xi_{1,1}-p+1$ ), and for $l=3, \ldots, p$, $2^{l-2}$ poles of the $l$-th generation (so that the total number of poles is $\left.1+\sum_{l=2}^{p} 2^{l-2}=2^{p-1}\right)$.

(2) For each interval $[-p-1,-p]$, the poles of one generation separate the set of poles of lower generations, and the zeros $\xi_{p, k}, k=1, \ldots, 2^{p-1}$, separate the set of all poles. That means that the set of poles of generation less than or equal to $l$ separate the zeros $\xi_{p, k}, k=1, \ldots, 2^{p-1}$, in groups of $2^{p-l}$ consecutive elements.

(3) For $l \geq 2$ the poles in $]-p-1,-p$ [ of the $l$-th generation are zeros of $f(z+p-l+1)$ but they are still poles of $f(z+j)$ if $0 \leq j \leq p-l$.

\section{Iteration of the rational function $\psi$}

In this section we will prove Theorem 1.2 and discuss the relationship with the classical study of iteration of rational functions of degree $\geq 2$, cf. e.g. [3].

We have already introduced the rational function $\psi$ by

$$
\psi(z)=z-\frac{1}{z}
$$

It is a mapping of $\mathrm{C} \backslash\{0\}$ onto $\mathrm{C}$ with a simple pole at $z=0$. Moreover, $\psi(0)=\psi(\infty)=\infty$. It is two-to-one with the exception that $\psi(z)= \pm 2 i$ has only one solution $z= \pm i$. It is strictly increasing on the half-lines ]- -0 [ and $] 0, \infty$ [, mapping each of them onto $R$. The functional equation (1.9) can be written

$$
f(z)=\psi(f(z+1)) .
$$

We notice that $\psi$ and hence all iterates $\psi^{\circ n}$ are Pick functions. It is convenient to define $\psi^{\circ 0}(z)=z$. We claim that the Julia set is $J(\psi)=\mathbf{R}^{*}$, and the Fatou set is $F(\psi)=\mathrm{C} \backslash \mathrm{R}$. This is because $\psi$ is conjugate to the rational function

$$
R(z)=\frac{3 z^{2}+1}{z^{2}+3}
$$

i.e. $g \circ R=\psi \circ g$, where $g$ is the Möbius transformation $g(z)=i(1+z) /(1-z)$. Note that $g$ is the Cayley transformation mapping the unit circle $T$ onto $\mathrm{R}^{*}$. In [3, p. 200] the Julia set of $R$ is determined as $J(R)=\mathrm{T}$, and the assertion follows. 
The sequence $\left(\lambda_{n}\right)_{n}$ is defined in terms of $\left(m_{n}\right)_{n}$ from (1.3) by

$$
\lambda_{0}=0, \quad \lambda_{n+1}=1 / m_{n}, \quad n \geq 0 .
$$

By (1.7) and (1.8) we clearly have

$$
m_{n}=F(n), \quad \lambda_{n}=f(n), \quad n \geq 0,
$$

hence by (3.2)

$$
\lambda_{n}=\psi\left(\lambda_{n+1}\right), \quad n \geq 0,
$$

which can be reformulated to

$$
\lambda_{n+1}=\frac{1}{2}\left(\lambda_{n}+\sqrt{\lambda_{n}^{2}+4}\right), \quad n \geq 0 .
$$

The following result is easy and the proof is left to the reader.

\section{Lemma 3.1. Defining}

$$
Y_{n}=\left(\psi^{\circ n}\right)^{-1}(\{0\})=\left\{z \in \mathrm{C} \mid \psi^{\circ n}(z)=0\right\},
$$

i.e.

$$
Y_{0}=\{0\}, \quad Y_{1}=\{-1,1\}, \quad Y_{2}=\{( \pm 1 \pm \sqrt{5}) / 2\}, \ldots
$$

we have for $n \geq 1$

(i) $\psi\left(Y_{n}\right)=Y_{n-1}, Y_{n}=\psi^{\circ-1}\left(Y_{n-1}\right)$,

(ii) The set of poles of $\psi^{\circ n}$ is $\cup_{j=0}^{n-1} Y_{j}$,

(iii) $Y_{n}$ consists of $2^{n}$ real numbers and is symmetric with respect to zero.

(iv) The function $\psi^{\circ}$ is strictly increasing from $-\infty$ to $\infty$ in each of the $2^{n}$ intervals in which $\cup_{j=0}^{n-1} Y_{j}$ divides $\mathrm{R}$. There is exactly one zero of $\psi^{\circ n}$ in each of these intervals, and these zeros form the set $Y_{n}$.

We write $Y_{n}=\left\{\alpha_{n, k}: k=1, \ldots, 2^{n}\right\}$ arranged in increasing order $(n \geq 1)$ :

$$
\alpha_{n, 1}<\alpha_{n, 2}<\cdots<\alpha_{n, 2^{n-1}}<0<\alpha_{n, 2^{n-1}+1}<\cdots<\alpha_{n, 2^{n}} .
$$

It is easy to see that $-\alpha_{n, 1}=\alpha_{n, 2^{n}}=\lambda_{n}$ for $n \geq 0$.

Proposition 3.2. The set

$$
\cup_{p=0}^{\infty} Y_{p}=\left\{\alpha_{p, k} \mid p \geq 0, k=1, \ldots, 2^{p}\right\}
$$

is dense in $\mathrm{R}$. 
Proof. The set in question is the so-called backward orbit of 0 for $\psi$, and since $0 \in J(\psi)$ the result follows by [3, Theorem 4.2.7].

We next give some asymptotic properties of the sequence $\left(\lambda_{n}\right)_{n}$ and the function $f$ :

LEMMA 3.3. (1) $\sqrt{n} \leq \lambda_{n} \leq \sqrt{2 n}, n \geq 0$.

(2) $\left(\lambda_{n}\right)_{n}$ is an increasing divergent sequence and $\lambda_{n+1} / \lambda_{n}$ is decreasing with $\lim _{n \rightarrow \infty} \frac{\lambda_{n+1}}{\lambda_{n}}=1$.

(3) $\lim _{n \rightarrow \infty}\left(\lambda_{n+1}^{2}-\lambda_{n}^{2}\right)=2$.

(4) $\lim _{n \rightarrow \infty} \frac{\lambda_{n}^{2}}{n}=2$.

(5) $\lim _{n \rightarrow \infty} \frac{\lambda_{n}^{2}-2 n}{\log n}=-\frac{1}{2}$.

(6) $\lim _{s \rightarrow \infty} f(s) / \sqrt{2 s}=1$.

(7) $\lim _{s \rightarrow \infty} f^{\prime}(s) \sqrt{2 s}=1$.

ProOF.

(1) These inequalities follow easily from (3.6) using induction on $n$.

(2) The sequence $\left(\lambda_{n}\right)_{n}$ increases to infinity since it is the reciprocal of the Hausdorff moment sequence $\left(m_{n}\right)_{n}$. By the Cauchy-Schwarz inequality $m_{n}^{2} \leq$ $m_{n-1} m_{n+1}$, which proves that $\left(\lambda_{n+1} / \lambda_{n}\right)_{n}$ is decreasing. The limit follows now easily from (3.6).

(3) Using (3.5) we can write

$$
\lambda_{n+1}^{2}-\lambda_{n}^{2}=\frac{\lambda_{n+1}+\lambda_{n}}{\lambda_{n+1}}=1+\frac{\lambda_{n}}{\lambda_{n+1}},
$$

and it suffices to apply part 2.

(4) is a consequence of part 3 and the following version of the Stolz criterion going back to [21]:

LEMMA 3.4. Let $\left(a_{n}\right)_{n},\left(b_{n}\right)_{n}$ be real sequences, where $\left(b_{n}\right)_{n}$ is strictly increasing tending to infinity. Then

$$
\lim _{n \rightarrow \infty} \frac{a_{n+1}-a_{n}}{b_{n+1}-b_{n}}=L \Rightarrow \lim _{n \rightarrow \infty} \frac{a_{n}}{b_{n}}=L .
$$


(5) follows by using again the Stolz criterion and taking into account that

$$
\begin{aligned}
\frac{\lambda_{n+1}^{2}-\lambda_{n}^{2}-2}{\log \frac{n+1}{n}} & =\frac{\lambda_{n+1}^{2}-\lambda_{n}^{2}-2 \lambda_{n+1}^{2}+2 \lambda_{n+1} \lambda_{n}}{\log \frac{n+1}{n}}=-\frac{\left(\lambda_{n+1}-\lambda_{n}\right)^{2}}{\log \frac{n+1}{n}} \\
& =-\frac{1}{n \log \frac{n+1}{n}} \frac{n}{\lambda_{n+1}^{2}} \rightarrow-\frac{1}{2}
\end{aligned}
$$

(6) Since $f$ is increasing and $f(n)=\lambda_{n}$, the assertion follows from part 4 .

(7) We write $f(n+1)-f(n)=f^{\prime}\left(t_{n}\right)$, for a certain $t_{n} \in(n, n+1)$. Since $f^{\prime}$ is decreasing $\left(f^{\prime}(s)\right.$ is completely monotonic), part 7 follows if we prove that $f^{\prime}\left(t_{n}\right) \sqrt{2 t_{n}}$ tends to 1 as $n$ tends to $\infty$. However, using the recursion formula for $\left(\lambda_{n}\right)_{n}$, we get

$$
f^{\prime}\left(t_{n}\right) \sqrt{2 t_{n}}=\left(\lambda_{n+1}-\lambda_{n}\right) \sqrt{2 t_{n}}=\frac{\sqrt{2(n+1)}}{\lambda_{n+1}} \frac{\sqrt{2 t_{n}}}{\sqrt{2(n+1)}},
$$

and it suffices to apply part 4 .

Proof of Theorem 1.2. We have already proved the properties (i) and (iii). To see (ii) we notice that $f=\mathscr{B}(\mu)$ is a Bernstein function, and therefore $1 / f$ is completely monotonic. Every completely monotonic function is logarithmically convex. For these statements see e.g. [10, § 14].

Suppose next that $\tilde{f}$ is a function satisfying (i)-(iii). Since $\tilde{f}(1)=1=\lambda_{1}$, we see by (iii) and (3.5) that $\tilde{f}(n)=\lambda_{n}$ for $n \geq 1$. Equation (1.11) is equivalent with

$$
\tilde{f}(s)=\lim _{n \rightarrow \infty} \psi^{\circ n}\left(\lambda_{n}\left(\frac{\lambda_{n+1}}{\lambda_{n}}\right)^{s}\right)
$$

and if we prove this equation for $0<s \leq 1$, then $\tilde{f}$ is uniquely determined on ]0,1] and hence by (iii) for all $s>0$.

We prove that the limit in (3.8) exists and coincides with $\tilde{f}(s)$ for $0<s \leq 1$. This is clear for $s=1$ since $\psi^{\circ n}\left(\lambda_{n+1}\right)=1$ for $n \geq 0$.

For any convex function $\phi$ on $] 0, \infty[$ we have for $0<s \leq 1$ and $n \geq 2$

$$
\phi(n)-\phi(n-1) \leq \frac{\phi(n+s)-\phi(n)}{s} \leq \phi(n+1)-\phi(n) .
$$

By taking $\phi=\log (1 / \tilde{f})$, which is convex by assumption, we get

$$
\log \frac{\lambda_{n-1}}{\lambda_{n}} \leq \frac{1}{s} \log \frac{\tilde{f}(n)}{\tilde{f}(n+s)} \leq \log \frac{\lambda_{n}}{\lambda_{n+1}}
$$


that is

$$
\left(\frac{\lambda_{n-1}}{\lambda_{n}}\right)^{s} \leq \frac{\lambda_{n}}{\tilde{f}(n+s)} \leq\left(\frac{\lambda_{n}}{\lambda_{n+1}}\right)^{s}
$$

which finally gives:

$$
\lambda_{n}\left(\frac{\lambda_{n+1}}{\lambda_{n}}\right)^{s} \leq \tilde{f}(n+s) \leq \lambda_{n}\left(\frac{\lambda_{n}}{\lambda_{n-1}}\right)^{s}, \quad 0<s<1 .
$$

Using that $\psi$ is increasing on $] 0, \infty\left[\right.$, we get by applying $\psi^{\circ n}$ to the previous inequality

$$
\psi^{\circ n}\left(b_{n}(s)\right) \leq \tilde{f}(s)=\psi^{\circ n}(\tilde{f}(n+s)) \leq \psi^{\circ n}\left(a_{n}(s)\right),
$$

where we have introduced

$$
a_{n}(s)=\lambda_{n}\left(\frac{\lambda_{n}}{\lambda_{n-1}}\right)^{s}, \quad b_{n}(s)=\lambda_{n}\left(\frac{\lambda_{n+1}}{\lambda_{n}}\right)^{s} .
$$

It is now enough to prove that

$$
\lim _{n \rightarrow \infty}\left(\psi^{\circ n}\left(a_{n}(s)\right)-\psi^{\circ n}\left(b_{n}(s)\right)=0 .\right.
$$

By applying the mean value theorem, we get for a certain $w \in] b_{n}(s), a_{n}(s)[$ that

$$
\begin{aligned}
\psi^{\circ n}\left(a_{n}(s)\right)-\psi^{\circ n}\left(b_{n}(s)\right) \\
\quad=\left(a_{n}(s)-b_{n}(s)\right)\left(\psi^{\circ n}\right)^{\prime}(w) \\
\quad=\left(a_{n}(s)-b_{n}(s)\right) \psi^{\prime}\left(\psi^{\circ n-1}(w)\right) \psi^{\prime}\left(\psi^{\circ n-2}(w)\right) \cdots \psi^{\prime}(w) .
\end{aligned}
$$

Since $\lambda_{n}<b_{n}(s)<w<a_{n}(s)$, we get $\lambda_{n-k}<\psi^{\circ k}\left(b_{n}(s)\right)<\psi^{\circ k}(w)$, $k=0,1, \ldots, n$, hence

$$
\begin{aligned}
\left|\psi^{\circ n}\left(a_{n}(s)\right)-\psi^{\circ n}\left(b_{n}(s)\right)\right| & \leq\left|a_{n}(s)-b_{n}(s)\right| \prod_{k=0}^{n-1}\left|\psi^{\prime}\left(\psi^{\circ k}(w)\right)\right| \\
& \leq\left|a_{n}(s)-b_{n}(s)\right| \prod_{k=0}^{n-1}\left(1+\frac{1}{\lambda_{n-k}^{2}}\right) \\
& =\lambda_{n}\left(\left(\frac{\lambda_{n}}{\lambda_{n-1}}\right)^{s}-\left(\frac{\lambda_{n+1}}{\lambda_{n}}\right)^{s}\right) \prod_{k=1}^{n}\left(1+\frac{1}{\lambda_{k}^{2}}\right)
\end{aligned}
$$




$$
\begin{aligned}
& \leq \lambda_{n}\left(\left(\frac{\lambda_{n}}{\lambda_{n-1}}\right)^{s}-\left(\frac{\lambda_{n+1}}{\lambda_{n}}\right)^{s}\right) \prod_{k=1}^{n}\left(1+\frac{1}{k}\right) \\
& =(n+1) \lambda_{n}\left(\left(\frac{\lambda_{n}}{\lambda_{n-1}}\right)^{s}-\left(\frac{\lambda_{n+1}}{\lambda_{n}}\right)^{s}\right),
\end{aligned}
$$

where we have used $\sqrt{k} \leq \lambda_{k}$ from Lemma 3.3 part 1 .

Using that $\left(x^{s}-y^{s}\right) \leq s(x-y)$ for $1<y<x$ and $0<s \leq 1$, we get

$$
\left|\psi^{\circ n}\left(a_{n}(s)\right)-\psi^{\circ n}\left(b_{n}(s)\right)\right| \leq s(n+1) \lambda_{n}\left(\frac{\lambda_{n}}{\lambda_{n-1}}-\frac{\lambda_{n+1}}{\lambda_{n}}\right),
$$

and by (3.6) we finally get

$$
\begin{aligned}
\mid \psi^{\circ n} & \left(a_{n}(s)\right)-\psi^{\circ n}\left(b_{n}(s)\right) \mid \\
\leq & \frac{1}{2} s(n+1) \lambda_{n}\left(\left(1+\sqrt{1+\frac{4}{\lambda_{n-1}^{2}}}\right)-\left(1+\sqrt{1+\frac{4}{\lambda_{n}^{2}}}\right)\right) \\
\quad= & \frac{1}{2} s(n+1) \lambda_{n}\left(\sqrt{1+\frac{4}{\lambda_{n-1}^{2}}}-\sqrt{1+\frac{4}{\lambda_{n}^{2}}}\right) \\
= & \frac{2 s(n+1) \lambda_{n}\left(\frac{1}{\lambda_{n-1}^{2}}-\frac{1}{\lambda_{n}^{2}}\right)}{\sqrt{1+\frac{4}{\lambda_{n-1}^{2}}}+\sqrt{1+\frac{4}{\lambda_{n}^{2}}}} \leq \frac{s(n+1)}{\lambda_{n} \lambda_{n-1}^{2}}\left(\lambda_{n}^{2}-\lambda_{n-1}^{2}\right),
\end{aligned}
$$

which tends to zero by part 2, 3 and 4 of Lemma 3.3.

For each real number $s$, we define the sequence $\left(\lambda_{n}(s)\right)_{n}$ by $\lambda_{0}(s)=s$ and

$$
\lambda_{n+1}(s)=\frac{\lambda_{n}(s)+\sqrt{\lambda_{n}(s)^{2}+4}}{2}, \quad n \geq 0 .
$$

Notice that $\lambda_{n+1}(s)$ is the positive root of $z^{2}-\lambda_{n}(s) z-1=0$ and that

$$
\psi\left(\lambda_{n+1}(s)\right)=\lambda_{n}(s) .
$$

Therefore, if $s \in Y_{l}$ then $\lambda_{n}(s) \in Y_{l+n}$, and for $s=0$ we have $\lambda_{n}(0)=\lambda_{n}$, $n \geq 0$. Furthermore, $\lambda_{n}\left(\lambda_{l}(s)\right)=\lambda_{n+l}(s)$.

Definition 3.5. For integers $k, l \geq 0$ we denote by $r(k, l)$ the unique solution $x \in\left\{1,2, \ldots, 2^{l}\right\}$ of the congruence equation $x \equiv k \bmod 2^{l}$.

Lemma 3.6. For $p \geq 1, k=1,2, \ldots, 2^{p}$ we have

(i) $\psi\left(\alpha_{p, k}\right)=\alpha_{p-1, r(k, p-1)}$. 
(ii) $\psi^{\circ l}\left(\alpha_{p, k}\right)=\alpha_{p-l, r(k, p-l)}$ for $l=0,1, \ldots, p$.

Proof. Since $\psi\left(Y_{p}\right)=Y_{p-1}$ and $\psi$ is strictly increasing mapping $]-\infty, 0[$ onto $\mathrm{R}$, we see that

$$
\psi\left(\alpha_{p, k}\right)=\alpha_{p-1, k}, \quad k=1,2, \ldots, 2^{p-1},
$$

and since similarly $\psi$ maps $] 0, \infty[$ onto $\mathrm{R}$ we get

$$
\psi\left(\alpha_{p, k}\right)=\alpha_{p-1, j}, \quad k=2^{p-1}+j, \quad j=1,2, \ldots, 2^{p-1} .
$$

In the first case $k=r(k, p-1)$ and in the second case $j=r(k, p-1)$ so the assertion (i) follows.

The assertion (ii) is clear for $l=0$ and $l=p$ and follows for $l=1$ by (i). Assuming (ii) for some $l$ such that $1 \leq l \leq p-2$ we get by (i)

$$
\psi^{\circ(l+1)}\left(\alpha_{p, k}\right)=\psi\left(\alpha_{p-l, r(k, p-l)}\right)=\alpha_{p-l-1, j},
$$

where $j:=r(r(k, p-l), p-l-1)$. By definition

$$
\begin{array}{ll}
k \equiv r(k, p-l) \bmod 2^{p-l}, & 1 \leq r(k, p-l) \leq 2^{p-l} \\
j \equiv r(k, p-l) \bmod 2^{p-l-1}, & 1 \leq j \leq 2^{p-l-1} .
\end{array}
$$

The first congruence also holds $\bmod 2^{p-l-1}$, hence $j \equiv k \bmod 2^{p-l-1}$ and finally $j=r(k, p-l-1)$.

COROLlary 3.7. For a zero $\xi_{p, k}$ of $f$ we have

(i) $f\left(\xi_{p, k}+l\right)=\alpha_{l, r(k, l)}, l=0,1, \ldots, p$,

(ii) $f\left(\xi_{p, k}+l\right)=\lambda_{l-p}\left(\alpha_{p, k}\right), l=p+1, p+2, \ldots$, where $\lambda_{n}(s)$ is defined in (3.9).

Proof. We first prove (i) for $l=p$, i.e. that $f\left(\xi_{p, k}+p\right)=\alpha_{p, k}$ since $r(k, p)=k$. Note that by (3.2) we have

$$
\psi^{\circ p}\left(f\left(\xi_{p, k}+p\right)\right)=f\left(\xi_{p, k}\right)=0
$$

hence $f\left(\xi_{p, k}+p\right) \in Y_{p}$. On the other hand $\left.\xi_{p, k}+p \in\right]-1,0[$, and since $f$ is strictly increasing satisfying $f(]-1,0[)=]-\infty, 0\left[\right.$, we see that $f\left(\xi_{p, k}+p\right)$, $k=1,2, \ldots, 2^{p-1}$ describe $2^{p-1}$ negative numbers in $Y_{p}$ in increasing order. Therefore, $f\left(\xi_{p, k}+p\right)=\alpha_{p, k}, k=1,2, \ldots, 2^{p-1}$.

By Lemma 3.6 and (3.2) we then get for $0 \leq l \leq p$

$$
f\left(\xi_{p, k}+l\right)=\psi^{\circ(p-l)}\left(f\left(\xi_{p, k}+p\right)\right)=\psi^{\circ(p-l)}\left(\alpha_{p, k}\right)=\alpha_{l, r(k, l)} .
$$


Clearly $0<f\left(\xi_{p, k}+p+1\right) \in Y_{p+1}$ and $\alpha_{p, k}=\psi\left(f\left(\xi_{p, k}+p+1\right)\right)$, hence $f\left(\xi_{p, k}+p+1\right)=\lambda_{1}\left(\alpha_{p, k}\right)$ by definition of $\lambda_{1}(s)$. The assertion (ii) follows easily by induction.

TheOREM 3.8. The numbers $\xi_{p, k}, \rho_{p, k}, p \geq 1, k=1, \ldots, 2^{p-1}$ and $\rho_{0}$ from Theorem 1.4 are given by the following formulas:

$$
\begin{aligned}
\xi_{p, k} & =\lim _{N \rightarrow \infty} \sqrt{2 N}\left(\sum_{l=1}^{p} \frac{1}{\alpha_{l, r(k, l)}}+\sum_{l=1}^{N-p} \frac{1}{\lambda_{l}\left(\alpha_{p, k}\right)}-\lambda_{N}\right) \\
\rho_{p, k} & =\prod_{l=1}^{p}\left(1+\frac{1}{\alpha_{l, r(k, l)}^{2}}\right)^{-1} \lim _{N \rightarrow \infty} \sqrt{2 N} \prod_{l=1}^{N}\left(1+\frac{1}{\lambda_{l}^{2}\left(\alpha_{p, k}\right)}\right)^{-1} \\
\rho_{0} & =\lim _{N \rightarrow \infty} \sqrt{2 N} \prod_{l=1}^{N}\left(1+\frac{1}{\lambda_{l}^{2}}\right)^{-1}
\end{aligned}
$$

Proof. By applying $N$ times the functional equation (1.9) for the function $f$ and using Corollary 3.7 , we have for $p<N$ :

$$
\begin{aligned}
0=f\left(\xi_{p, k}\right) & =f\left(\xi_{p, k}+N\right)-\sum_{l=1}^{N} \frac{1}{f\left(\xi_{p, k}+l\right)} \\
& =f\left(\xi_{p, k}+N\right)-\left(\sum_{l=1}^{p} \frac{1}{\alpha_{l, r(k, l)}}+\sum_{l=1}^{N-p} \frac{1}{\lambda_{l}\left(\alpha_{p, k}\right)}\right) .
\end{aligned}
$$

Writing

$$
y_{N, p, k}=\sum_{l=1}^{p} \frac{1}{\alpha_{l, r(k, l)}}+\sum_{l=1}^{N-p} \frac{1}{\lambda_{l}\left(\alpha_{p, k}\right)},
$$

we get $f\left(\xi_{p, k}+N\right)=y_{N, p, k}$. For $N \rightarrow \infty$ it follows by part 6 of Lemma 3.3 that $y_{N, p, k} \sim \sqrt{2 N}$. Since $f$ is a strictly increasing bijection of $(-1,+\infty)$ onto R, we can consider its inverse $f^{-1}$. Then we have $N=f^{-1}\left(\lambda_{N}\right)$, hence $\xi_{p, k}=f^{-1}\left(y_{N, p, k}\right)-f^{-1}\left(\lambda_{N}\right)$. Since $\xi_{p, k}$ is negative and $f$ is increasing, we deduce that $y_{N, p, k}<\lambda_{N}$. This gives for a certain number $\left.\sigma_{N, p, k} \in\right] y_{N, p, k}, \lambda_{N}[$ that

$$
\begin{aligned}
\xi_{p, k}=f^{-1}\left(y_{N, p, k}\right)-f^{-1}\left(\lambda_{N}\right) & =\left(f^{-1}\right)^{\prime}\left(\sigma_{N, p, k}\right)\left(y_{N, p, k}-\lambda_{N}\right) \\
& =\frac{y_{N, p, k}-\lambda_{N}}{f^{\prime}\left(\eta_{N, p, k}\right)},
\end{aligned}
$$


where we have written $\eta_{N, p, k}=f^{-1}\left(\sigma_{N, p, k}\right)$. Clearly $\left.\eta_{N, p, k} \in\right] \xi_{p, k}+N, N[$.

Taking into account that $\lim _{s \rightarrow \infty} f^{\prime}(s) \sqrt{2 s}=1$ (part 7 of Lemma 3.3), we have

$$
\xi_{p, k}=\lim _{N} \sqrt{2 N}\left(y_{N, p, k}-\lambda_{N}\right),
$$

that is, (3.11) holds.

The number $f^{\prime}\left(\xi_{p, k}\right)$ can be computed as follows: Deriving the functional equation (1.9) for $f$, we get

$$
f^{\prime}(z)=f^{\prime}(z+1)\left(1+\frac{1}{f^{2}(z+1)}\right)
$$

hence by iteration

$$
f^{\prime}(z)=f^{\prime}(z+N) \prod_{l=1}^{N}\left(1+\frac{1}{f^{2}(z+l)}\right) .
$$

Using Corollary 3.7 and $\lim _{s \rightarrow \infty} f^{\prime}(s) \sqrt{2 s}=1$, (Lemma 3.3, part 7) we get for $z=\xi_{p, k}$

$$
f^{\prime}\left(\xi_{p, k}\right)=\prod_{l=1}^{p}\left(1+\frac{1}{\alpha_{l, r(k, l)}^{2}}\right) \lim _{N \rightarrow \infty} \frac{1}{\sqrt{2 N}} \prod_{l=1}^{N}\left(1+\frac{1}{\lambda_{l}^{2}\left(\alpha_{p, k}\right)}\right),
$$

and since $\rho_{p, k}=1 / f^{\prime}\left(\xi_{p, k}\right)$ by (1.12), we see that (3.12) holds.

Applying (3.14) for $z=0$, we get

$$
f^{\prime}(0)=f^{\prime}(N) \prod_{l=1}^{N}\left(1+\frac{1}{\lambda_{l}^{2}}\right),
$$

and (3.13) follows by (1.12) and $\lim _{N \rightarrow \infty} f^{\prime}(N) \sqrt{2 N}=1$.

We give some values of the numbers of Theorem 3.8:

\begin{tabular}{cc}
\hline$\rho_{0}=0.68 \ldots$ & $\xi_{0}=0$ \\
\hline$\rho_{1,1}=0.14 \ldots$ & $\xi_{1,1}=-1.46 \ldots$ \\
$\rho_{2,1}=0.06 \ldots$ & $\xi_{2,1}=-2.61 \ldots$ \\
$\rho_{2,2}=0.05 \ldots$ & $\xi_{2,2}=-2.33 \ldots$ \\
\hline
\end{tabular}

THeOREM 3.9. The density $\mathscr{D}$ given by (1.15) satisfies

$$
\mathscr{D}(t) \sim \frac{1}{\sqrt{2 \pi(1-t)}} \quad \text { for } t \rightarrow 1
$$


Proof. By formula (1.8) and Lemma 3.3 part 6 we get

$$
F(s)=\int_{0}^{1} t^{s} \mathscr{D}(t) d t \sim \frac{1}{\sqrt{2 s}}, \quad s \rightarrow \infty,
$$

or

$$
\int_{0}^{\infty} e^{-u s} \mathscr{D}\left(e^{-u}\right) e^{-u} d u \sim \frac{1}{\sqrt{2 s}}, \quad s \rightarrow \infty .
$$

By the Karamata Tauberian theorem, cf. [12, Theorem 1.7.1'], we get

$$
\int_{0}^{t} \mathscr{D}\left(e^{-u}\right) e^{-u} d u \sim \sqrt{\frac{2 t}{\pi}}, \quad t \rightarrow 0,
$$

and since $\mathscr{D}$ is increasing we can use the Monotone Density theorem, cf. [12, Theorem 1.7.2b], to conclude that

$$
\mathscr{D}\left(e^{-u}\right) e^{-u} \sim \frac{1}{\sqrt{2 \pi u}}, \quad u \rightarrow 0,
$$

which is equivalent to the assertion.

\section{Miscellaneous about the fixed point}

The fixed point sequence $\left(m_{n}\right)_{n}$ given by (1.3) satisfies $m_{n+1}=\Phi\left(m_{n}\right)$ with

$$
\Phi(x)=\frac{\sqrt{4 x^{2}+1}-1}{2 x}, \quad x>0 .
$$

This makes it possible to express $\left(m_{n}\right)_{n}$ as iterates of $\Phi$, viz.

$$
m_{n}=\Phi^{\circ n}(1) .
$$

From Lemma 3.3 part 4 we get the asymptotic behaviour of $m_{n}$ as

$$
m_{n} \sim \frac{1}{\sqrt{2 n}}, \quad n \rightarrow \infty .
$$

This behaviour can also be deduced from a general result about iteration, cf. [13, p. 175]. The authors want to thank Bruce Reznick for this reference as well as the following description of $\left(m_{n}\right)_{n}$.

Proposition 4.1. Define $\left.\left.h_{n} \in\right] 0, \pi / 4\right]$ by $\tan h_{n}=m_{n}$ and let

$$
G(x)=\frac{1}{2} \arctan (2 \tan x), \quad|x|<\frac{\pi}{2} .
$$


Then

$$
h_{n}=G^{\circ n}\left(\frac{\pi}{4}\right)
$$

Proof. We have

$$
\tan h_{n}=m_{n}=\frac{m_{n+1}}{1-m_{n+1}^{2}}=\frac{\tan h_{n+1}}{1-\tan ^{2} h_{n+1}}=\frac{1}{2} \tan \left(2 h_{n+1}\right),
$$

hence $h_{n+1}=G\left(h_{n}\right)$ and the assertion follows.

A Hausdorff moment sequence $\left(a_{n}\right)_{n}$ is called infinitely divisible if $\left(a_{n}^{\alpha}\right)_{n}$ is a Hausdorff moment sequence for all $\alpha>0$. If $a_{n}=\int_{0}^{1} t^{n} d v(t), n \geq 0$ then $\left(a_{n}\right)_{n}$ is infinitely divisible if and only if $v$ is infinitely divisible for the product convolution $\tau \diamond v$ of measures $[0, \infty[$ defined by

$$
\int g d \tau \diamond v=\iint g(s t) d \tau(s) d v(t) .
$$

For a general study of these concepts see [22], [5], [6]. In case the measure $v$ does not charge 0 , the notion is the classical infinite divisibility on the locally compact group $] 0, \infty[$ under multiplication.

Proposition 4.2. Hausdorff moment sequences of the form (1.1) are infinitely divisible.

Proof. Let $v \neq 0$ be a positive measure on $[0,1]$ and let $a_{n}=\int t^{n} d v(t)$, $n \geq 0$ be the corresponding Hausdorff moment sequence. Let $\alpha>0$ be fixed. We shall prove that $\left(\left(a_{0}+a_{1}+\cdots+a_{n}\right)^{-\alpha}\right)_{n}$ is a Hausdorff moment sequence.

For $0<c<1$ we denote by $v_{c}=v \mid\left[0, c\left[+v(\{1\}) \delta_{c}\right.\right.$, where the first term denotes the restriction of $v$ to $\left[0, c\left[\right.\right.$. Then $\lim _{c \rightarrow 1} v_{c}=v$ weakly and in particular for each $n \geq 0$

$$
a_{n}(c):=\int_{0}^{1} t^{n} d v_{c}(t) \rightarrow a_{n} \quad \text { for } \quad c \rightarrow 1 .
$$

It therefore suffices to prove that

$$
\left(\left(a_{0}(c)+a_{1}(c)+\cdots+a_{n}(c)\right)^{-\alpha}\right)_{n}
$$

is a Hausdorff moment sequence. By a simple calculation we find

$$
\begin{aligned}
\left(\sum_{k=0}^{n} a_{k}(c)\right)^{-\alpha} & =\left(\int_{0}^{1} \frac{1-t^{n+1}}{1-t} d v_{c}(t)\right)^{-\alpha} \\
& =\left(\int_{0}^{1} \frac{d v_{c}(t)}{1-t}-\int_{0}^{1} t^{n} \frac{t d v_{c}(t)}{1-t}\right)^{-\alpha}=H\left(\tau_{n}\right),
\end{aligned}
$$


where

$$
\tau_{n}=\int_{0}^{1} t^{n} \frac{t d v_{c}(t)}{1-t}, \quad H(z)=\left(\int_{0}^{1} \frac{d v_{c}(t)}{1-t}-z\right)^{-\alpha} .
$$

The function $H$ is clearly holomorphic in

$$
|z|<\int_{0}^{1} \frac{d v_{c}(t)}{1-t}
$$

with non-negative coefficients in the power series. Applying Lemma 2.1 in [9], shows that (4.1) is a Hausdorff moment sequence.

COROllary 4.3. The fixed point sequence $\left(m_{n}\right)_{n}$ is infinitely divisible.

REMARK 4.4. By Corollary 4.3 the fixed point measure $\mu$ is infinitely divisible for the product convolution. The image measure $\eta$ of $\mu$ under $\log (1 / t)$ is an infinitely divisible probability measure in the ordinary sense, because $\log (1 / t)$ maps products to sums. The measure $\eta$ has the density

$$
\mathscr{D}\left(e^{-u}\right) e^{-u}=\rho_{0} e^{-u}+\sum_{p=1}^{\infty} \sum_{k=1}^{2^{p-1}} \rho_{p, k} e^{-u\left(1-\xi_{p, k}\right)}, \quad u>0
$$

with respect to Lebesgue measure on the half-line. Since (4.2) is clearly a completely monotonic density, the infinite divisibility of $\eta$ is also a consequence of the Goldie-Steutel theorem, see [20, Theorem 10.7]. These remarks also show that Corollary 4.3 can be inferred from the complete monotonicity of (4.2) via the Goldie-Steutel theorem. The formula

$$
\int_{0}^{\infty} e^{-u s} d \eta(u)=\int_{0}^{1} t^{s} d \mu(t)=F(s)=e^{-\log f(s+1)}, \quad s \geq 0
$$

shows that $\log f(s+1)$ is the Bernstein function associated with the convolution semigroup $\left(\eta_{t}\right)_{t>0}$ of probability measures on the half-line such that $\eta_{1}=\eta$, see $[10$, p. 68$]$.

Remark 4.5. Let $\mathscr{H}_{I}$ denote the set of normalized infinitely divisible Hausdorff moment sequences. By Proposition 4.2 we have $T(\mathscr{H}) \subseteq \mathscr{H}_{I}$. We claim that this inclusion is proper. In fact, it is easy to see that $T: \mathscr{H} \rightarrow T(\mathscr{H})$ is one-to-one, and that

$$
T^{-1}(\mathbf{b})_{n}=\frac{1}{b_{n}}-\frac{1}{b_{n-1}}, \quad n \geq 1,
$$


for $\mathbf{b}=\left(b_{n}\right)_{n} \in T(\mathscr{H})$. It follows that

$$
T(\mathscr{H})=\left\{\mathbf{b} \in \mathscr{H} \mid\left(\frac{1}{b_{n}}-\frac{1}{b_{n-1}}\right)_{n} \in \mathscr{H}\right\} .
$$

(Here $1 / b_{n}-1 / b_{n-1}=1$ for $n=0$.) Then $\mathbf{b} \in \mathscr{H}_{I} \backslash T(\mathscr{H})$ if we define $b_{n}=1 /(n+1)^{2}$.

The functions $f, F$ being holomorphic in $\operatorname{Re} z>-1$ with a pole at $z=-1$, they have power series expansions

$$
F(z)=1+\sum_{n=1}^{\infty} a_{n} z^{n}, \quad f(z)=\sum_{n=1}^{\infty} b_{n} z^{n}, \quad|z|<1,
$$

and the radius of convergence is 1 for both series.

Proposition 4.6. The coefficients in (4.3) are given for $n \geq 1$ by

$$
\begin{aligned}
a_{n} & =\frac{1}{n !} \int_{0}^{1}(\log t)^{n} d \mu(t)=(-1)^{n}\left(\rho_{0}+\sum_{p=1}^{\infty} \sum_{k=1}^{2^{p-1}} \frac{\rho_{p, k}}{\left(1-\xi_{p, k}\right)^{n+1}}\right), \\
b_{n} & =-\frac{1}{n !} \int_{0}^{1} \frac{(\log t)^{n}}{1-t} d \mu(t) \\
& =(-1)^{n-1}\left(\rho_{0} \zeta(n+1,0)+\sum_{p=1}^{\infty} \sum_{k=1}^{2^{p-1}} \rho_{p, k} \zeta\left(n+1,-\xi_{p, k}\right)\right),
\end{aligned}
$$

where

$$
\zeta(s, a)=\sum_{n=1}^{\infty} \frac{1}{(n+a)^{s}}, \quad s>1, a>-1
$$

is the Hurwitz zeta function.

Proof. The formula for $a_{n}$ follows from (1.7) and (1.13), and the formula for $b_{n}$ follows from (1.6) and (1.14).

AcKnowledgement. The authors wish to thank Henrik L. Pedersen for help with producing the graph of $f$.

\section{REFERENCES}

1. Akhiezer, N. I., The Classical Moment Problem, Oliver and Boyd, Edinburgh, 1965.

2. Artin, E., The Gamma Function, Holt, Rinehart and Winston, New York, 1964.

3. Beardon, A. F., Iteration of Rational Functions, Grad. Texts in Math. 132 (1991). 
4. Berg, C., Quelques remarques sur le cône de Stieltjes, in: Séminaire de Théorie du potentiel, Paris n.5, Lecture Notes in Math. 814 (1980).

5. Berg, C., On powers of Stieltjes moment sequences, I, J. Theoret. Probab. 18 No. 4 (2005), 871-889.

6. Berg, C., On powers of Stieltjes moment sequences, II, J. Comput. Appl. Math. 199 (2007), 23-38.

7. Berg, C., Christensen, J. P. R., and Ressel, P., Harmonic Analysis on Semigroups. Theory of Positive Definite and Related Functions, Grad. Texts in Math. 100 (1984).

8. Berg, C., Durán, A. J., A transformation from Hausdorff to Stieltjes moment sequences, Ark. Mat. 42 (2004), 239-257.

9. Berg, C., Durán, A. J., Some transformations of Hausdorff moment sequences and harmonic numbers, Canad. J. Math. 57 (2005), 941-960.

10. Berg, C., Forst, G., Potential Theory on Locally Compact Abelian Groups, Ergeb. Math. Grenzgeb. 87 (1975).

11. Bertoin, J., Lévy Processes, Cambridge University Press, Cambridge, 1996.

12. Bingham, N. H., Goldie, C. M., and Teugels, J. L., Regular Variation, Cambridge University Press, Cambridge, 1987.

13. De Bruijn, N. G., Asymptotic Methods in Analysis, North-Holland Publishing Company, Amsterdam, 1958.

14. Diaconis, P., Freedman, D., The Markov moment problem and de Finetti's theorem. I, Math. Z. 247 (2004), 183-199.

15. Diaconis, P., Freedman, D., The Markov moment problem and de Finetti's theorem. II, Math. Z. 247 (2004), 201-212.

16. Donoghue Jr., W. F., Monotone Matrix Functions and Analytic Continuation, Springer-Verlag, Berlin-Heidelberg-New York, 1974.

17. Hausdorff, F., Momentenprobleme für ein endliches Intervall, Math. Z. 16 (1923), 220-248.

18. Itô, M., Sur les cônes convexes de Riesz et les noyaux complètement sous-harmoniques, Nagoya Math. J. 55 (1974), 111-144.

19. Reuter, G. E. H., Über eine Volterrasche Integralgleichung mit totalmonotonem Kern, Arch. Math. 7 (1956), 59-66.

20. Steutel, F. W., Van Harn, K., Infinite Divisibility of Probability Distributions on the Real Line, Marcel Dekker, Inc., New York, Basel 2004.

21. Stolz, O., Ueber die Grenzwerthe der Quotienten, Math. Annalen 14 (1878), 231-240.

22. Tyan, S., The structure of Bivariate distribution functions and their relation to Markov processes, $\mathrm{Ph}$. D. Thesis, Princeton University 1975.

23. Widder, D. V., The Laplace Transform, Princeton University Press, Princeton, 1941.

INSTITUT FOR MATEMATISKE FAG

KØBENHAVNS UNIVERSITET

UNIVERSITETSPARKEN 5

DK-2100 KØBENHAVN Ø

DENMARK

E-mail: berg@math.ku.dk
DEPARTAMENTO DE ANÁLISIS MATEMÁTICO UNIVERSIDAD DE SEVILLA

APDO (P.O. BOX) 1160

41080 SEVILLA

SPAIN

E-mail: duran@us.es 